\title{
O Vínculo Droga Valvopatia
}

\author{
Max G rinberg, A Ifredo Halpern, Eduardo G iusti Rossi, Cláudia Cozer Leite, J oão Cesar N unes Sbano
}

São Paulo, SP

Ação de droga não é fator etiopatogênico habitualmente cogitado perante valvopatia adquirida. Não obstante, efeitos adversos de remédios anti-enxaqueca ${ }^{1-5} \mathrm{e}$ anorexígenos ${ }^{6,7}$ estão vinculados a casos de cardiopatia valvar, alguns inclusive submetidos à correção cirúrgica.

\section{Derivados do ergot}

Metisergida $^{2-5,8}$ - Nas décadas de 60/70, o derivado do ergot metisergida era o mais eficaz profilático para enxaqueca. Na ocasião, estimava-se o consumo em 500 mil pessoas.

Em 1964, John Graham, então diretor da Headache Research Foundations, em Boston, foi alertado sobre a ausculta de sopros de insuficiência mitral ou aórtica durante uso contínuo da droga.

Uma primeira avaliação, compreendendo 27 pacientes com casos de fibrose retroperitonial induzida pela metisergida, revelou sete (26\%) casos com sopro cardíaco. Em período de dois anos, o pesquisador pôde observar o desenvolvimento sem outra causa aparente de sopro cardíaco expressivo em 16 de seus pacientes.

Dez anos após a constatação original, revisão publicada pelo mesmo Serviço incluiu não mais do que 48 casos de vínculo da droga com sopro cardíaco.

Este número restrito de casos foi suficiente para incorporar as seguintes observações ao conhecimento sobre cardiopatia valvar: 1) taxa de 3,6\% dos pacientes sob uso continuado de metisergida desenvolve sopro cardíaco. Devemos conjecturar que lesões valvares subclínicas, atualmente identificáveis pela ecocardiografia, possam ter ficado à margem desta proporção; 2) a maioria dos pacientes não apresentava sopro cardíaco previamente ao uso; 3) não havia outra causa de sopro cardíaco; 4) a intensidade do sopro cardíaco mostrou-se inicialmente crescente; 5) a suspensão da droga associou-se à regressão total $(46 \%)$ ou parcial (12\%) do sopro cardíaco em $58 \%$ dos casos; 6) os sopros reveladores de estenose e/ou insuficiência tinham origem nas valvas mitral, aórtica e tricúspide; 7) sopro cardíaco superpôs-se em $26 \%$ ao desenvolvimento de fibrose retroperitonial, condição também, potencialmente, reversí-

Instituto do Coração do Hospital das Clínicas - FMUSP

Correspondência: Max Grinberg - Incor - Av. Dr. Enéas C. Aguiar, 44 - 05403-000 - São Paulo, SP

Recebido para publicação em 3/12/97

Aceito em 4/2/98 vel com a suspensão da droga; 8) o exame anatomopatológico das valvas que vieram a ser operadas ou estudadas à necropsia revelou características similares entre si de comprometimento de folhetos valvares, cordas tendíneas e músculos papilares; 9) o aspecto patológico fundamental é um processo fibroproliferativo, que se desenvolve como uma camada de revestimento de tecido colágeno. Não há destruição da estrutura valvar subjacente. Este aspecto o diferencia da doença reumática; 10) o processo fibrótico da valva aórtica pode se estender para a parede ventricular esquerda, aorta e óstio de artéria coronária (causa documentada de infarto do miocárdio).

A orientação clínica para uso da metisergida passou a se restringir a: 1) formas graves de enxaqueca; 2) pacientes confiáveis para cumprir rigorosamente a orientação de suspender, periodicamente, o medicamento e se submeter a exames de controle; 3 ) acréscimo das seguintes contra-indicações às vigentes: lesões valvares, descompensação cardíaca de qualquer causa, doenças associadas a estados de fibrose.

Estima-se que 1000-1200 pacientes estejam sob uso de metisergida, atualmente, no Brasil, droga praticamente reservada para casos de cefaléia em salva.

Ergotamina ${ }^{1,3}$ - Registros semelhantes de sopros cardíacos e de lesões valvares resultaram do uso de ergotamina.

A contribuição diagnóstica da ecocardiografia mostrou-se valiosa através da identificação de espessamentos, doming diastólico, imobilidade e perda de coaptação de cúspides mitral e tricúspide, espessamentos e encurtamentos de corda tendínea mitral e espessamento e retração de válvula aórtica. Diferentes graus de insuficiência valvar auscultados foram confirmados.

\section{Relação com a serotonina}

Este vínculo farmacovalvar parece ser a semelhança química destas drogas com a serotonina. A metisergida compete por alguns de seus receptores, funcionando, na maioria das vezes, como agente antiserotonina; contudo, há também evidências que ela seja agonista da serotonina no sistema cardiovascular ${ }^{9}$. A ergotamina, por sua vez, tem múltiplas propriedades que incluem bloqueio alfa-adrenérgico, antagonismo à serotonina, e estímulo direto de vasos periféricos e cerebrais.

Admite-se que a serotonina cause estímulo direto do 
crescimento de fibroblastos, muito embora estudos sobre o efeito da metisergida sobre fibroblastos, usando cultura de tecido mitral não tenham confirmado esta hipótese ${ }^{5}$.

A ligação da valvopatia aos alcalóides do ergot através da serotonina é reforçada pela ampliação das analogias para o âmbito da síndrome do carcinóide. As lesões carcinóides, que em cerca de $80 \%$ dos casos localizam-se no intestino, associam-se à cardiopatia valvar em $50 \%$ dos casos: insuficiência tricúspide associada ou não a estenose, em praticamente todos, insuficiência/estenose pulmonar, na maioria, e comprometimento mitral, por vezes com de aórtica, em menos do que $10 \%$ dos casos ${ }^{10,11}$. Os achados morfológicos (ecocardiográficos e anatomopatológicos) superpõem-se aos descritos com as referidas drogas. A exposição do endocárdio a níveis elevados de serotonina é a admitida etiopatogenia para a cardiopatia do carcinóide, assim fechando o circuito de semelhanças.

\section{Anorexígenos}

Fenfluramina + fentermina (fen-phen) ${ }^{6}$ - A possível associação entre lesões valvares e anorexígenos foi cogitada recentemente pelas observações reunidas por Connolly e col ${ }^{6}$ sobre a mistura de fenfluramina com fentermina, conhecida como fen-phen.

A fenfluramina, utilizada há 35 anos, é uma amina simpaticomimética, que atua estimulando a produção e diminuindo a recaptação de serotonina. Em conseqüência, promove elevação dos níveis deste neurotransmissor em determinados locais do sistema nervoso central, particularmente, na região ventro-medial do hipotálamo. O efeito terapêutico é a sensação de saciedade e, portanto, diminuição de ingestão de alimentos ${ }^{12}$.

O seu d-isômero dexfenfluramina é um produto sintetizado mais recentemente (existe há 12 anos na França, sete no Brasil e há quase 2 nos Estados Unidos da América (EUA)).

Já a fentermina (anorético não utilizado no Brasil, mas relacionado ao fenproporex e à anfepramona, aqui existentes) age através da noradrenalina, e talvez da dopamina; atua no hipotálamo e determina diminuição da sensação de fome.

$\mathrm{O}$ fen-phen vem sendo prescrito há vários anos nos EUA; estima-se que 18 milhões de pessoas devem tê-lo usado até 1996.

\begin{tabular}{|lcc|}
\hline \multicolumn{4}{|c|}{ Tabela I - Dados gerais referentes aos $\mathbf{5 7}$ pacientes estudados } \\
\hline & ECO (-) & ECO (+) \\
\hline Número de casos & $30(53 \%)$ & $27(47 \%)$ \\
Média etária (anos) & $30(23-58)$ & $47(33-64)$ \\
Percentual de mulheres & 86 & 82 \\
Média de peso corpóreo (kg) & $82(54-142)$ & $84(54-127)$ \\
Média de altura (m) & $1,62(1,50-1,79)$ & $1,62(1,48-1,78)$ \\
Percentual de fumantes & 11 & 20 \\
Tempo de uso da droga (meses) & $10,9(6-28)$ & $11,8(6-33)$ \\
Associação com outros anorexígenos (\%) & 50 & 40 \\
\hline $\begin{array}{l}\text { ECO (-) ausência de anormalidades passíveis de serem relacionadas ao uso da } \\
\text { droga; ECO (+) presença de anormalidade passíveis de serem relacionadas ao } \\
\text { uso da droga. }\end{array}$ & \\
\hline
\end{tabular}

\begin{tabular}{|l|}
\hline \multicolumn{2}{|c|}{$\begin{array}{c}\text { Tabela II - Dados ecocardiográficos dos pacientes com alterações } \\
\text { atribuíveis ao uso da dexfenfluramina }\end{array}$} \\
\hline
\end{tabular}

Foi deste universo que Connolly e col ${ }^{6}$ identificaram 24 mulheres, com média etária de 44 anos, que desenvolveram valvopatia em graus variáveis, inclusive aquele determinante de tratamento cirúrgico $(21 \%)$, após média de 12 meses de tratamento.

Esta inexpressiva taxa numérica de $0,000013 \%$ foi o bastante para a observação ter sido julgada de alta relevância no âmbito da saúde pública e divulgada imediatamente para a comunidade médica dois meses antes da publicação do artigo.

Como conseqüência, quando esta se deu em agosto de 1997, o Federal Drug Agency, através do seu programa MedWatch, que tem como um dos objetivos facilitar a notificação de reações adversas de remédios, tinha recebido mais 61 notificações de valvopatia associada ao "fenphen", além de outros 12 referentes a uso isolado de fenfluramina ou dexfenfluramina.

As cinco valvas mitrais operadas (duas submetidas à plástica e três à substituição), uma aórtica, concomitantemente excisada e uma tricúspide reparada mostraram os folhetos revestidos por um material branco brilhante, que prejudicava a coaptação; a histologia revelou que essa verdadeira placa consistia de miofibroblastos proliferando em matriz extracelular exuberante.

Dados de ausculta cardíaca e ecocardiográficos diagnosticaram combinações de sopros de insuficiência mitral/ aórtica/tricúspide e imagens peculiares em todos os casos.

Os dados clínicos e morfológicos remeteram os observadores imediatamente ao vínculo entre as drogas, que agem via serotonina e as alterações valvulares cardíacas descritas com os derivados do ergot.

\section{Nossas observações}

Os dados referentes à avaliação ecodopplercardiográfica que realizamos em 57 pacientes sob uso de dexfenfluramina estão nas tabelas I e II.

Esta agressão de valva cardíaca durante farmacoterapia com fenfluramina suscita reflexões e reaviva ensinamentos esquecidos ou extraviados no torvelinho da multiplicidade de informações da literatura: 1) o risco estimado de 18 por 1 milhão de usuários/ano de ocorrência de hipertensão pulmonar primária pelo uso de fenfluramina, associado a $45 \%$ de mortalidade ao fim de quatro anos, não 
impediu sua comercialização; um registro com taxa menor de morbidade, envolvendo situação de manifestação clínica mais explícita, foi suficiente para, imediatamente, colocar a droga fora de uso; 2) foram precisos vários anos de consumo para que se desse o reconhecimento deste efeito colateral cardiológico; 3 ) mutualidade de desapontamento médico-paciente, pois a baixa freqüência dos habituais desconfortos droga-dependentes no uso continuado da droga, de certa forma, presumia lua-de-mel prolongada; 4) os mecanismos de doença seguem certos padrões e o vínculo droga-valvopatia apresenta um modelo aparentemente uniforme; 5) as analogias etiopatogênicas e de expressão clínica permitem exercitar suposições evolutivas, ou seja, a experiência adquirida com os derivados do ergot poderia ser transposta para a fenfluramina; 6) as conjecturas tornam-se um método desejável, na medida em que aspectos éticos dificultam estudos prospectivos; 7) nesta linha de pensamento, fica a perspectiva de a fenfluramina e a dexfenfluramina serem reservadas, como opção, para quando houver real benefício ao prognóstico de estados mórbidos associados à obesidade; 8) na eventualidade de uso, dever-seia considerar a menor dose eficaz e suspensão a intervalos de tempo; 9) considerar contra-indicação ao uso qualquer anomalia valvar já existente ou o paciente não confiável para aderir a uma estratégia de acompanhamento; 10) obrigatoriedade de exame clínico-ecocardiográfico prévio para a caracterização do estado cardiológico de identidade; programação de revisões periódicas.

\section{Referências}

1. Redfield MM, Nicholson WJ, Edwards WD, Tajik AJ - Valve disease associated with ergot alkaloid use: echocardiographic and pathologic correlations. Ann Intern Med 1992; 117: 50-2

2. Mason JW, Billingham ME, Friedman JP- Methysergide-induced heart disease. A case of multivalvular and myocardial fibrosis. Circulation 1977; 56: 889-90.

3. Hauck AJ, Edwards WD, Danielson GK, Mullany CJ, Bresnahan DR - Mitral and aortic valve disease associated with ergotamine therapy for migraine. Arch Pathol Lab Med 1990; 114: 62-4.

4. Graham JR - Cardiac and pulmonary fibrosis during methysergide therapy for headache. Am J Med Sci 1967; 254: 23-34.

5. BanaDS, MacNealPS,LeComptePM,Shah Y, GrahamJR-Cardiac murmurs andendocardial fibrosis associated with methysergide therapy. Am Heart J 1974; 88: 640-55.

6. Connolly HM, Crary JL, McGoon MD et al - Valvular heart disease associated with fenfluramine-phentermine. N Engl J Med 1997; 337: 581-8.
7. Cannistra LB, Davis SM, Bauman AG - Valvular heart disease associated with dexfenfluramine. NEngl J Med 1997; 337: 636

8. Slugg PH, Kunkel RS - Complications of methysergide therapy: retroperitoneal fibrosis, mitral regurgitation, edema and hemolytic anemia. JAMA 1970; 213 : 297-8.

9. Fanciullacci M, Granchi G, Sicuteri F- Ergotamine and methysergide as serotonin partial agonists in migraine. Headache 1976; 28: 64-70.

10. Roberts WC, Sjoerdsma A - The cardiac disease associated with the carcinoid syndrome (carcinoid heart disease). Am J Med 1964; 36: 5-34.

11. Pellikka PA, Tajik AJ, Khandheria BK et al - Carcinoid heart disease. Clinical and echocardiographic spectrum in 74 patients. Circulation 1993; 87: 1188-96.

12. Weintraub M, Hasday JD, Mushlin AI, Lockwood DH - A double-blind clinical trial in weight control: use of fenfluramine and phentemine alone and in combination. Arch Intern Med 1984; 144: 1143-8. 